\title{
Ions Slow Water Dynamics at Non-Ionic Surfactant Interfaces
}

\author{
Christopher P. Baryiames*, Emily Ma†, and Carlos R. Baiz* \\ *Department of Chemistry, University of Texas at Austin, 105 East 24th Street, Stop A5300, \\ Austin, Texas 78712-1224, United States; *cbaiz@cm.utexas.edu \\ †Department of Chemistry, Northwestern University, 2145 Sheridan Road, Evanston, IL 60208, \\ United States
}

Number of Pages: 8

Number of Figures: 4

Number of Tables: $\mathbf{3}$

Table of Contents:

S1. SAMPLE PREPARATION AND CHARACTERIZATION

Table S1: Dynamic light scattering size characterization of reverse micelles

\section{S2. FTIR MEASUREMENTS}

\section{S3. 2D IR MEASUREMENTS}

Figure S1: 2D IR spectra of salt-free reverse micelles

Figure S2: 2D IR spectra of $\mathrm{NaCl}$-containing reverse micelles

Figure S3: 2D IR spectra of $\mathrm{CaCl}_{2}$-containing reverse micelles

\section{S4. MOLECULAR DYNAMICS SIMULATIONS}

Table S2: Three-component FFCF fitting

Table S3: Simulated hydrogen bond populations

Table S4: Origins of simulated hydrogen bonds

Figure S4: $\mathrm{C}=\mathrm{O}$ bond orientational populations

\section{S1: Sample Preparation \& Characterization}

Sorbitan monostearate was purchased from Alfa Aesar (Haverhill, MA) and purified using flash chromatography as described in our previous work. ${ }^{1}$ In brief, a 65:25:2-by-volume mixture of chloroform, methanol, and water was used as an eluent and silica was used as the standing phase. The purity of each fraction was assessed using LC-MS and those fractions with $>80 \%$ sorbitan monostearate were combined to give a sample of $84 \%$ purity. The majority impurity is sorbitan distearate (10\%), with tri- and tetrastearates present in small quantity (6\% total). 
We prepared $30 \mathrm{mM}$ solutions of purified surfactant in octane, which were stored over molecular sieves to remove residual water. Surfactants were dissolved in sufficient $\mathrm{D} 2 \mathrm{O}$ or $\mathrm{D} 2 \mathrm{O} / \mathrm{NaCl}$ or $\mathrm{D} 2 \mathrm{O} / \mathrm{CaCl}_{2}$ solution to maintain a $22: 1$ water:surfactant mole ratio, then sonicated at $45^{\circ} \mathrm{C}$ for 5 minutes. RMs were characterized by DLS and found to be monodisperse regardless of the composition of the aqueous phase. Salt solutions were prepared to $2 \mathrm{M}$ concentration of ions in $\mathrm{D}_{2} \mathrm{O}$. The critical micelle concentration of sorbitan surfactants is given in the literature as 1-3 mM. ${ }^{2}$ This means that in our samples, $\sim 3-10 \%$ of surfactant molecules may be dissolved in the oil phase without participating in the microemulsion structure. This amount is insufficient to significantly alter the experimental lineshapes.

Table S1: Dynamic light scattering size characterization of reverse micelles

\begin{tabular}{c|c|c} 
System & Diameter $(\mathbf{n m})$ & PDI Width \\
\hline $\mathrm{No} \mathrm{Salt}$ & $143 \pm 3$ & 0.205 \\
$\mathrm{NaCl}$ & $135 \pm 4$ & 0.198 \\
$\mathrm{CaCl}_{2}$ & $141 \pm 5$ & 0.237
\end{tabular}

\section{S2: FTIR measurements}

Infrared absorption spectra were collected using a Bruker Vertex 70 instrument at $1 \mathrm{~cm}^{-1}$ wavenumber resolution. 32 scans were used to generate each spectrum. Samples were held between two $\mathrm{CaF}_{2}$ windows with a $50 \mu \mathrm{m}$ spacer. We collected temperature-controlled spectra from $35^{\circ} \mathrm{C}$ to $75^{\circ} \mathrm{C}$ in $3^{\circ} \mathrm{C}$ steps to ensure the sample was continuously in the RM phase by avoiding the transition temperature at $\sim 30^{\circ} \mathrm{C}$. Temperatures were set using a recirculating chiller with an accuracy of $0.1^{\circ} \mathrm{C}$.

Hydrogen bond populations were extracted using our previously-described Gaussian fitting method. Thermodynamics were calculated using the same van't Hoff analysis as described in the aforementioned study. ${ }^{1}$

\section{S3: 2D IR Measurements}

Ultrafast 2D IR spectra were collected using a custom-built setup as described in our previous work. ${ }^{3}$ Briefly, a sequence of three mid-IR laser pulses, centered around $5.7 \mu \mathrm{m}$, interact with the sample. The delay between the first pair of pulses is Fourier transformed to generate the excitation frequency axis $\left(\omega_{1}\right)$. The probe is a pulse $\sim 200 \mathrm{~cm}^{-1}$ in width, which is measured in a grating spectrometer equipped with an MCT array to define the detection axis $\left(\omega_{3}\right)$. Measuring spectra at different waiting time delays, ranging from $150 \mathrm{fs}$ to $3 \mathrm{ps}$, allows us to extract the picosecond spectral diffusion of the OHB peak, as it is the most intense and therefore best resolved in the spectrum. We use the center line slope method to quantify spectral diffusion, 
fitting the resulting slopes to an exponential decay with a bootstrapping method described previously. ${ }^{1,4}$

Salt-Free 2D IR spectra
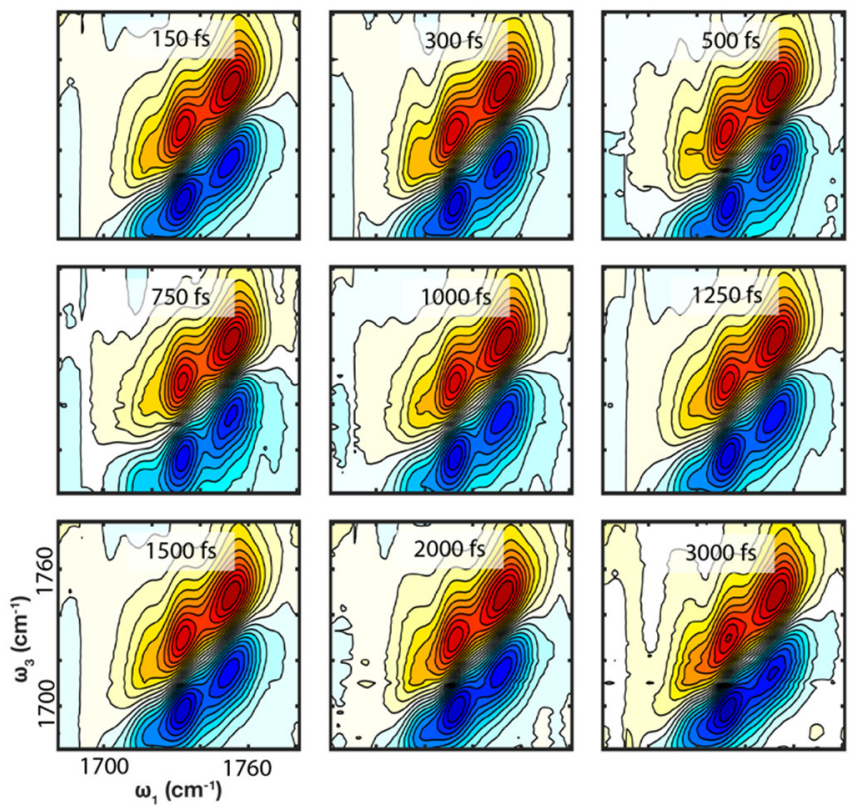

Figure S1: Experimental carbonyl stretch 2D IR spectra of salt-free surfactants in reverse micelles 


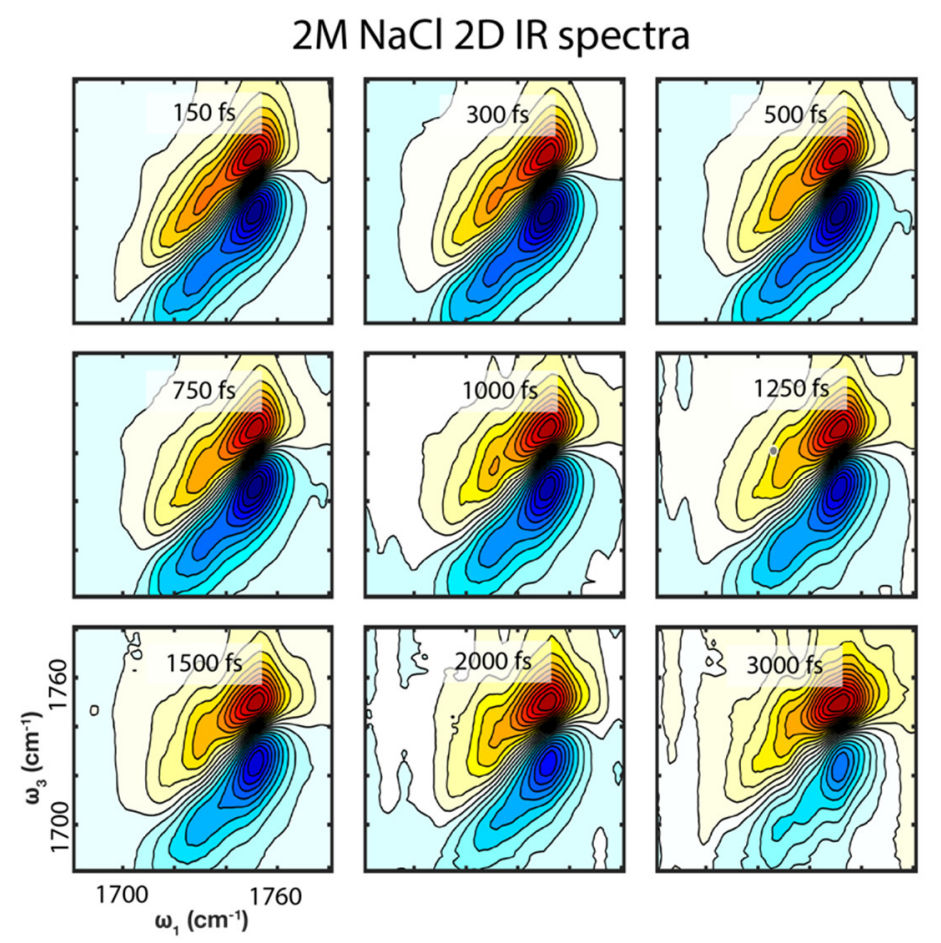

Figure S2: Experimental carbonyl stretch 2D IR spectra of surfactants in reverse micelles with $2 \mathrm{M} \mathrm{NaCl}$ in the aqueous phase

$2 \mathrm{M} \mathrm{CaCl}_{2} 2 \mathrm{D}$ IR spectra
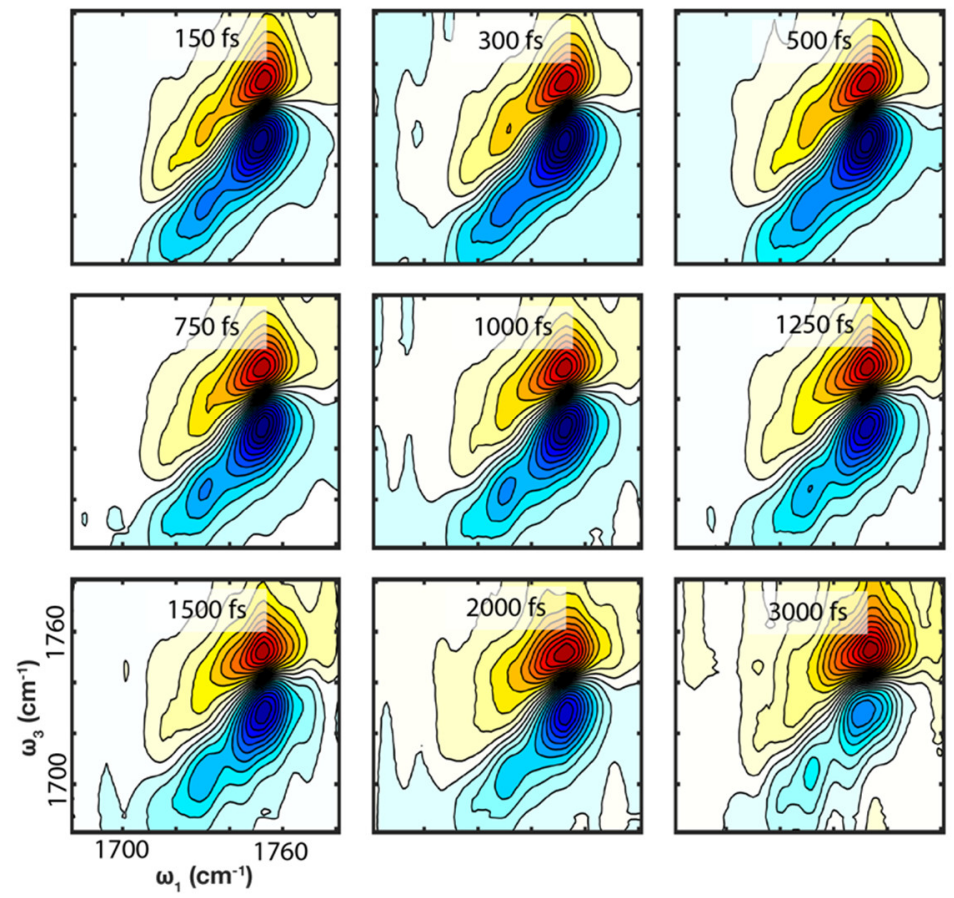

Figure S3: Experimental carbonyl stretch 2D IR spectra of surfactants in reverse micelles with $2 \mathrm{M} \mathrm{CaCl}_{2}$ in the aqueous phase 


\section{S4: Molecular Dynamics Simulations}

Atomistic molecular dynamics (MD) simulations were carried out using the GROMACS 5.04 package of programs. ${ }^{5}$ Initial structures and CHARMM36 topologies were generated using the CHARMM-GUI ligand reader and modeler, with the exception of water. ${ }^{6}$ The TIP3P water model was used. Boxes were prepared using Packmol and detergent molecules were packed to an areaper-surfactant of $35 \AA^{2}$. Each box is comprised of 92 soribitan monostearate molecules, 2000 water molecules, and 420 octane molecules. For systems containing ions, two cations and the corresponding number of chloride counterions to maintain a neutral charge were added to the box using the gmx genion GROMACS functionality. This functionality replaces water molecules with ions, resulting in $\mathrm{NaCl}$-containing boxes having 1996 water molecules and $\mathrm{CaCl}_{2}$-contining boxes having 1994 waters. All systems were energy minimized, then equilibrated in the NVT and NPT ensembles for 24 ns each. Production runs of 24 ns were used as this has been previously been shown to be sufficient for these systems. ${ }^{1}$ When changing the force field parameters for the ions, the system was allowed to re-equilibrate for 200ns in the NPT ensemble.

Frequency-frequency correlation functions (FFCF) were computed using a frequency map approach previously described. ${ }^{1}$ In brief, the electric field at each ester group is computed and used as the basis for an electrostatic frequency map previously developed in our group. ${ }^{1,4,7}$ These analyses were performed on $10 \mathrm{~ns}$ trajectories, sampled every $20 \mathrm{fs}$. The FFCF is fit to a sum of three exponentials: a fast component ( $\sim 50 \mathrm{fs}$ ), a middle component ( $1 \mathrm{ps})$, and a very slow component ( $\sim 10 \mathrm{ps})$. The $\sim 1 \mathrm{ps}$ decay occurs on the same timescale as dynamics observed from 2D IR experiments, so this is the component the analysis is focused around. The fast and slow components cannot be measured using our experimental 2D IR setup.

Table S2: Timescales from three-component fits to FFCF decays.

\begin{tabular}{c|c|c|c} 
System & Fast Component (ps) & $\begin{array}{c}\text { Intermediate } \\
\text { Component (ps) }\end{array}$ & $\begin{array}{c}\text { Slow Component } \\
\text { (ps) }\end{array}$ \\
\hline $\mathrm{No} \mathrm{Salt}$ & $0.06 \pm 0.02$ & $0.7 \pm 0.2$ & $13.0 \pm 0.1$ \\
$\mathrm{NaCl}$ & $0.12 \pm 0.04$ & $0.9 \pm 0.1$ & $7.1 \pm 0.3$ \\
$\mathrm{CaCl}_{2}$ & $0.16 \pm 0.05$ & $1.6 \pm 0.4$ & $7.7 \pm 0.3$
\end{tabular}

Interfacial hydrogen bond populations are extracted using gmx hbond GROMACS tool with the standard geometric criterion ( $3.5 \AA$ length and $35^{\circ}$ angle cutoffs) using the full production MD trajectories. Hydrogen bonds were computed from $\mathrm{C}=\mathrm{O}$ groups to water molecules as well as hydroxyl groups on detergent molecules. In all cases, HB populations were within standard deviation of each other. Likewise, the presence of ions did not effect the ratio of HBs to water molecules and HBs to neighboring detergents, further indicating that the experimental results are driven primarily by changes in $\mathrm{C}=\mathrm{O}$ orientation alone. 
Table S3: Hydrogen bond populations extracted from triplicate MD production runs.

\begin{tabular}{c|c|c|c} 
System & OHB & 1HB & 2HB \\
\hline $\mathrm{No} \mathrm{Salt}$ & $62 \pm 4 \%$ & $33 \pm 2 \%$ & $14 \pm 2 \%$ \\
$\mathrm{NaCl}$ & $60 \pm 3 \%$ & $37 \pm 5 \%$ & $13 \pm 3 \%$ \\
$\mathrm{CaCl}_{2}$ & $63 \pm 4 \%$ & $32 \pm 4 \%$ & $14 \pm 3 \%$
\end{tabular}

Ester carbonyl orientational populations were computed by calculating the angle between the $\mathrm{C}=\mathrm{O}$ bond dipole and the interface normal for each $\mathrm{C}=\mathrm{O}$ bond in a 100 ps trajectory, sampled every $20 \mathrm{fs}$. $\mathrm{C}=\mathrm{Os}$ were again divided into ion-adjacent $(<2 \mathrm{~nm}$ apart) and ion-distant $(>2 \mathrm{~nm}$ apart). The angles of all ion-distant carbonyls were collected, so as to compare salt-containing and salt-free boxes. These angles are then plotted as a histogram. The histogram is then fit to a combination of three Gaussian functions, representing outward-, perpendicular, and inwardfacing carbonyls. The Gaussians were integrated and their fractional areas are used to estimate the percentage of carbonyls in each orientational environment. Error bars were determined by multiplying the relative peak area by the fraction of the peak that is overlapped by neighboring populations. Thus, the greater the overlap between peaks, the greater the uncertainty in its contribution to the overall histogram. Peaks were fit without constraints on width or amplitude. Centers were constrained from $180-120^{\circ}$ for inward-facing $C=0 s, 120-60^{\circ}$ for perpendicular $C=O s$, and $60-0^{\circ}$ for outward-facing $\mathrm{C}=\mathrm{Os}$. Inward-facing groups have their dipoles oriented towards the alkyl region of the micelle, outward-facing COs are oriented towards the aqueous phase, and perpendicular carbonyls are oriented along the interface.

Table S4: Origins of simulated hydrogen bonds extracted from triplicate MD

\begin{tabular}{c|c|c} 
System & HBs to Water & HBs to Surfactant \\
\hline $\mathrm{No} \mathrm{Salt}$ & $17 \pm 3 \%$ & $31 \pm 4 \%$ \\
$\mathrm{NaCl}$ & $18 \pm 2 \%$ & $33 \pm 5 \%$ \\
$\mathrm{CaCl}_{2}$ & $17 \pm 2 \%$ & $32 \pm 4 \%$
\end{tabular}




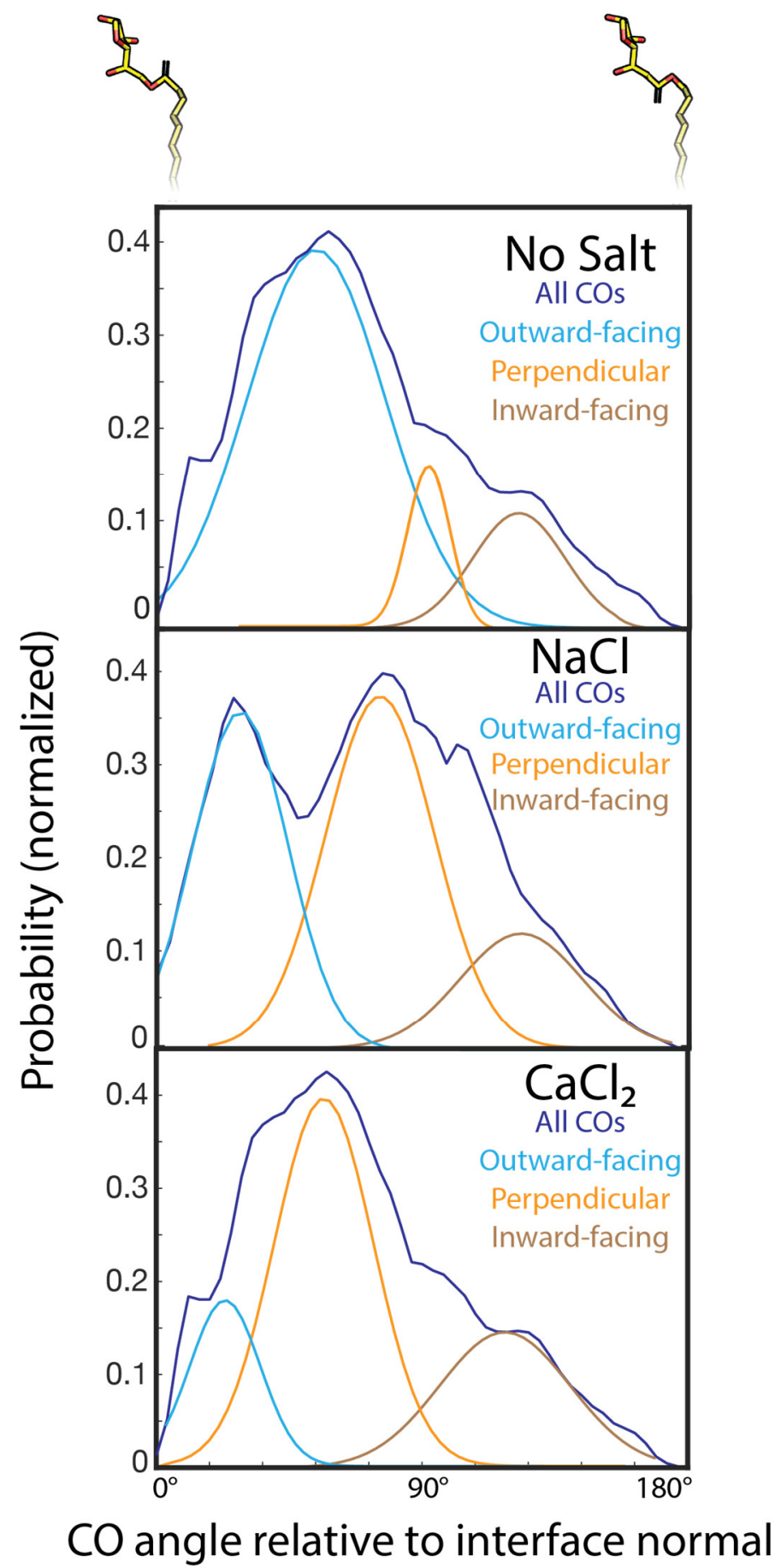

Figure S4: Histograms of carbonyl group angles relative to the interface normal and the corresponding three-component fits used to determine angle populations. Cartoons above the plots represent molecular structures corresponding to the $0^{\circ}$ and $180^{\circ}$ populations. Outwardfacing refers to carbonyl pointing towards the aqueous side of the interface, whereas inwardfacing refers to carbonyls pointing towards the oleic side. 


\section{References}

(1) Baryiames, C. P.; Teel, M.; Baiz, C. R. Interfacial H-Bond Dynamics in Reverse Micelles: The Role of Surfactant Heterogeneity. Langmuir 2019, 35 (35), 11463-11470. https://doi.org/10.1021/acs.langmuir.9b01693.

(2) Owusu Apenten, R. K.; Zhu, Q.-H. Interfacial Parameters for Selected Spans and Tweens at the Hydrocarbon -Water Interface. Food Hydrocoll. 1996, 10 (1), 27-30. https://doi.org/10.1016/S0268-005X(96)80050-6.

(3) Edington, S. C.; Gonzalez, A.; Middendorf, T. R.; Halling, D. B.; Aldrich, R. W.; Baiz, C. R. Coordination to Lanthanide Ions Distorts Binding Site Conformation in Calmodulin. Proc. Natl. Acad. Sci. 2018, 115 (14), E3126-E3134. https://doi.org/10.1073/pnas.1722042115.

(4) Baryiames, C. P.; Baiz, C. R. Slow Oil, Slow Water: Long-Range Dynamic Coupling across a Liquid-Liquid Interface. J. Am. Chem. Soc. 2020, 142 (18), 8063-8067. https://doi.org/10.1021/jacs.0c00817.

(5) Berendsen, H. J. C.; van der Spoel, D.; van Drunen, R. GROMACS: A Message-Passing Parallel Molecular Dynamics Implementation. Comput. Phys. Commun. 1995, 91 (1), 4356. https://doi.org/10.1016/0010-4655(95)00042-E.

(6) Brooks, B. R.; Brooks, C. L.; MacKerell, A. D.; Nilsson, L.; Petrella, R. J.; Roux, B.; Won, Y.; Archontis, G.; Bartels, C.; Boresch, S.; Caflisch, A.; Caves, L.; Cui, Q.; Dinner, A. R.; Feig, M.; Fischer, S.; Gao, J.; Hodoscek, M.; Im, W.; Kuczera, K.; Lazaridis, T.; Ma, J.; Ovchinnikov, V.; Paci, E.; Pastor, R. W.; Post, C. B.; Pu, J. Z.; Schaefer, M.; Tidor, B.; Venable, R. M.; Woodcock, H. L.; Wu, X.; Yang, W.; York, D. M.; Karplus, M. CHARMM: The Biomolecular Simulation Program. J. Comput. Chem. 2009, 30 (10), 1545-1614. https://doi.org/10.1002/jcc.21287.

(7) Edington, S. C.; Flanagan, J. C.; Baiz, C. R. An Empirical IR Frequency Map for Ester C=O Stretching Vibrations. J. Phys. Chem. A 2016, 120 (22), 3888-3896. https://doi.org/10.1021/acs.jpca.6b02887. 